\title{
Journal of Clinical Education in Physical Therapy - growth during COVID
}

$\mathrm{T}$ he Journal of Clinical Education in Physical Therapy (JCEPT) mission emphasizes facilitating development of and disseminating evidence related to clinical education in physical therapy. Our distinguished editorial board members continue to provide guidance as we develop, while representing various clinical specialties, research interests, and roles in physical therapy education.

The coronavirus (COVID) pandemic has posed serious challenges for everyone including personal health and financial crises, educational adaptation to online learning, and professional research challenges. Interest in JCEPT has continued to grow, nevertheless. Submissions and published papers for the 2021 volume have doubled. As an open-access journal, JCEPT is developing an international reach; papers last year were downloaded about 1,000 times with $>15 \%$ of users located beyond the United States. In the current volume, we look forward to papers with timely topics: impact of integrated clinical experiences on physical therapy student clinical performance, student mental health during clinical education, competency of clinical instructors, entry level caseload expectations during terminal clinical experiences, and student physical therapist burnout and grit during terminal clinical education experiences. Many of the opportunities and challenges we face along the continuum of learning is not unique to physical therapy. In an effort to integrate the views of other professions, we are pleased to publish our first resident case study in our Inter-Professional Corner.

Physical therapy education programs create learning experiences in a controlled environment to approximate authentic clinical situations. There is, however, no substitute for clinical education where learners apply knowledge and skills in real clinical practice. Clinical instructors (CIs) are the primary educators during entry level Doctor of Physical Therapy (DPT) clinical education experiences which account for approximately one third of the DPT curriculum. ${ }^{1}$ While typical characteristics and qualities of CIs have been identified, ${ }^{2-4}$ there is wide variation in the quality of clinical education that DPT students experience. One reason for variations in clinical education quality appears to be CIs clinical teaching skills. ${ }^{2}$ The National Study of Excellence and Innovation in Physical Therapy Education has suggested the establishment of a standard comprehensive, longitudinal approach for performance-based learning outcomes across the learner continuum. ${ }^{5}$ Competencies, or characteristics of an individual, are an example of a defined outcome.

In the current volume, Bilyeu et al. describe a path to the stepwise development of competencies for CIs to help prepare them to teach. This information can help develop personalized professional development for CIs that could reduce the variation in clinical teaching. The increase in new and expanding DPT programs has increased demand for clinical education placements. Bayliss introduces a re-designed clinical education curricular model that emphasizes in-class patient experiences along with integrated clinical experiences as an alternate solution to meet this growing challenge. In addition, increased productivity pressures add to the challenges of CIs and students. Sherman et al. examine expected student caseloads across and within clinical settings, while Kish et al. explore student burnout and grit within clinical settings. Finally, Eubanks et al. explores the experiences of the Director of Clinical Education (DCE) that often expand beyond the recommended roles ${ }^{6}$ to include supporting the student during mental health challenges. ${ }^{7}$

The growth of JCEPT has been exciting to see and we look forward to developing further as other manuscripts progress through the publication pathway. JCEPT accepts original research, narrative and systematic reviews, innovative teaching methods or educational models relevant to clinical training, clinical education or clinical case reports, and exceptional critically appraised topics all of which can address any element of physical therapy education related to clinical practice.

Christopher Kevin Wong and Jean Fitzpatrick Timmerberg Editors in Chief 


\section{References}

1. Commission on Accreditation in Physical Therapy Education. Aggregate program data: 2019-2020 Physical therapist education programs fact sheet. Available from: https://www. capteonline.org/globalassets/capte-docs/aggregate-data/20192020-aggregate-pt-program-data.pdf [cited 29 August 2021].

2. Recker-Hughes C, Wetherbee E, Buccieri KM, et al. Essential characteristics of quality clinical education experiences: Standards to facilitate student learning. J Phys Ther Educ (2014) 28: 48-55. doi: 10.1097/00001416-201400001-00009

3. McCallum CA, Reed R, Bachman S, et al. A systematic review of physical therapist clinical instructor demographics and key characteristics: Impact on student clinical education experiences. J Phys Ther Educ (2016) 30: 11-20. doi: 10.1097/00001416-201630030-00004
4. Mostrom E. What makes a good clinical teacher? In: Jensen GM, Mostrom E, eds. Handbook of teaching and learning for physical therapists, 3rd ed. St. Louis, MO: Elsevier/Butterworth-Heinemann; 2013, pp. 159-82.

5. Jensen GM, Nordstrom T, Mostrom E, et al. National study of excellence and innovation in physical therapist education: Part 1-design, method, and results. Phys Ther (2017) 97: 857-74. doi: 10.1093/ptj/pzx061

6. Erickson M, Birkmeier M, Booth M et al. Recommendations from the common terminology panel of the American Council of Academic Physical Therapy, Phys Ther (2018) 98(9): 754-62. doi: $10.1093 / \mathrm{ptj} / \mathrm{pzy} 075$

7. Evans TM, Bira L, Gastelum JB, et al. Evidence for a mental health crisis in graduate education. Nat Biotechnol (2018) 36(3): 282-84. doi: 10.1038/nbt.4089 